\title{
Fortbildung
}

Schweiz Z Ganzheitsmed 2010;22:342-343

DOI: 10.1159/000322247

\section{Internationaler Kongress für biologische Lasertherapie und Akupunktur, 25.-27. Juni 2010}

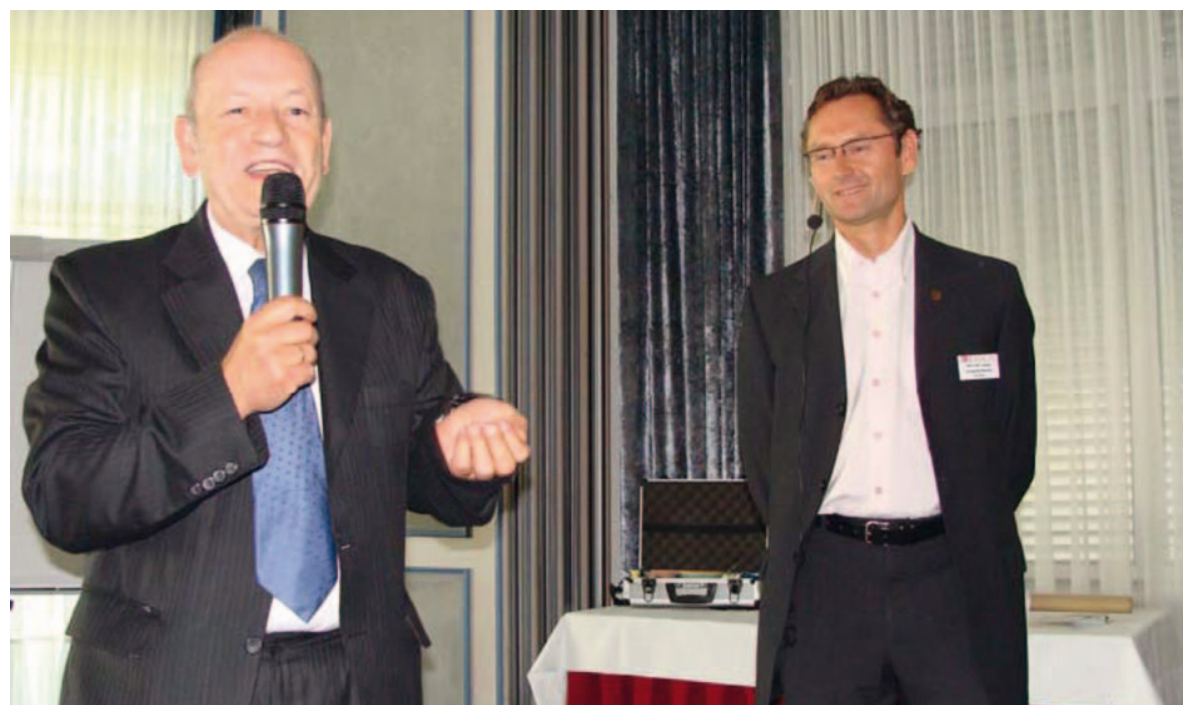

Herr Dr. Heinrich aus Berlin referierte über die intravenöse Sauerstofftherapie, die von vielen Therapeuten heute mit grossem Erfolg in Kombination mit der intravenösen Lasertherapie bei vielen chronischen Erkrankungen eingesetzt wird. Dabei werden insbesondere Augenerkrankungen (Makuladegeneration) erfolgreich behandelt.

Erstmalig war ein Referent aus Tschechien angereist. Dr. Rusek berichtete über Lasernadelakupunktur und intravenöse Laserblutbestrahlung bei chirurgischen Indikationen. Er stellte ausgesprochen interessante neue Ansätze in der Behandlung von Analfissuren und Hämorrhoiden vor.

Den nächsten Vortrag hielt Heilpraktiker Klaus Kleiber, der seine Praxis in einem Eifelkloster hat. Er berichtete über Akupunktur und intravenöse Lasertherapien bei $\mathrm{Au}$ generkrankungen, insbesondere bei Makuladegeneration und Glaukom.
Er hat dazu ein eigenes Behandlungsschema entwickelt.

Unser langjähriger Referent, Herr Dr. Andreas Wirz-Ridolfi aus Reinach in der Schweiz, referierte über Blockaden als Therapiehindernisse bei Lasertherapie und Akupunktur und wie man diese überwinden kann.

Damit war das Programm am ersten Kongresstag zu Ende und es folgte am Abend eine wunderschöne Dampferfahrt auf der Weser mit Musik und Tanz und unserer Band «Jenny und Dean» aus Dubai.

Am zweiten Kongresstag eröffnete Herr Professor Litscher von der Universität Graz das Vortragsprogramm mit einem hochinteressanten Beitrag über die Weiterentwicklung der modernen Lasernadelakupunktur und stellte wichtige neuartige Untersuchungen mittels Blaulaserbehandlung vor.

Als nächster Referent berichtete Steve Liu aus Tucson in Arizona, USA, (bisheriger Präsident der «North von ATP.

(๑) 2010 S. Karger GmbH, Freiburg
Dr. med. Dipl.-Chem. Michael Weber

1. Vorsitzender EGLA (Europäische Gesellschaft für biologische Lasertherapie und Akupunktur), Klinik Neu-Mariahilf

Humboldtallee 10-12, 37073 Göttingen, Deutschland

Tel. +49 551-50429660, Fax -50429669

info@weberinstitut.de 


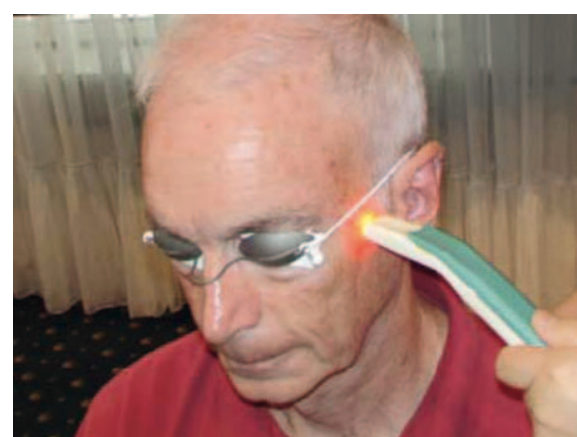

American Association for Lasertherapy») über integrative Ansätze bei chronischen Weichteilschäden und -überlastungen.

Im nächsten Vortrag stellte Dr. Dorfer, Präsident der österreichischen Gesellschaft für kontrollierte Akupunktur, sehr schöne und interessante Fälle von Allergiebehandlungen bei Kindern mittels Laserakupunktur vor.

Dr. Michael Grandjean, Lehrbeauftragter für Akupunktur der Universität Frankfurt, stellte die Weiterentwicklung seiner abdominellen Akupunkturmethode vor und demonstrierte einen Laser, mit dem man das Punktesystem auf den Bauch projizieren kann. Die abdominelle Akupunktur hat sich als neuartiges Mikrosystem als ausserordentlich effektiv erwiesen.

Im nächsten Vortrag berichtete Professor Peter Dorsher von der Mayo Clinic in Jacksonville, Florida, USA, über neuroanatomische Grundlagen der Akupunktur für Gelenk- und Muskelschmerzen. Er zeigte auf, dass die chinesischen Akupunkturpunkte in einem grossen Prozentsatz mit speziellen Nervenaussichtspunkten identisch sind.

Anschliessend demonstrierte Professor Chung, Leiter der Hahnemann's Memorial Clinic in Taipeh, Taiwan, interessante therapeutische Behandlungsmöglichkeiten der Fibromyalgie. Er hat dazu auch ein eigenes Buch geschrieben, das über die EGLA bestellt werden kann.

Im nächsten Vortrag berichtete Professor Latif Aftab von der Universitätsklinik Lahore in Pakistan über die Behandlung von diabetischen
Fussulzera mittels intravenöser Lasertechnik und zeigte ausgezeichnete Ergebnisse bei dieser Indikation.

In einem hochinteressanten Vortrag berichtete Frau Professor Naeser von der Universität Boston, USA, über erfolgreiche transkranielle LED-Therapie bei Patienten mit traumatischen Gehirnverletzungen, welche zu erheblich verbesserten kognitiven Eigenschaften führte. Sie erklärte dies anhand von mehreren Fallberichten.

Dr. Peter Aluani, Mitglied des Vorstandes der «Österreichischen Gesellschaft für Kontrollierte Akupunktur», demonstrierte viele verschiedene Möglichkeiten, Laserakupunktur und intravenöse Blutlasertherapie bei chronischen Schmerzsyndromen erfolgreich zu kombinieren.

Frau Professor Edith Perez, Leiterin der Brustkrebsabteilung an der Mayo Clinic in Jacksonville, Florida, USA, hielt einen exzellenten Vortrag über die derzeitigen schulmedizinischen Behandlungsmethoden und zeigte sich sehr offen dafür, an neuen Studien mit Einsatz der modernen Lasertechnik mitzuarbeiten.

Am Nachmittag wurde das Programm mittels vertiefender Workshops zu den entsprechenden Themen abgerundet und endete am Abend schliesslich mit einer wunderschönen Veranstaltung auf der Burgruine Plesse mit einem Livekonzert von «Jenny und Dean» mit Barbecue und Tanz.

Am letzten Kongresstag referierte zunächst Professor Köstler, Präsident der Österreichischen Gesellschaft für Onkologie, über gemeinsame Eigenschaften von Krebszellen und die sich daraus ergebenden therapeutischen

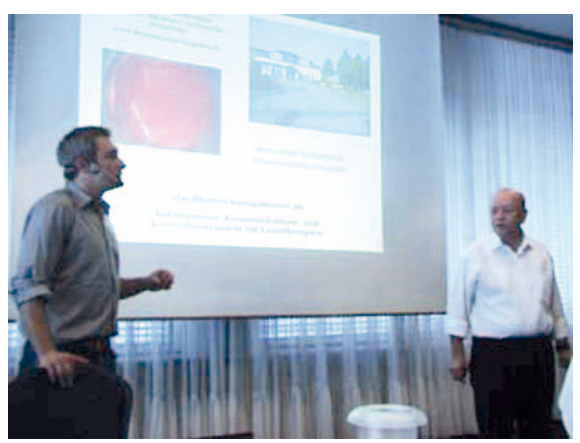

Optionen, insbesondere auch im Rahmen von alternativen Heilverfahren.

Dr. Giesen, Internist und Endoskopiespezialist (Darmzentrum Südwestfalen) aus Wenden bei Olpe, referierte über das spannende Gebiet der lokalen und systemischen photodynamischen Therapie bei Speiseröhren- und Blasenkrebs.

Im nächsten Vortrag berichtete Dr. Mary Dyson aus London, England, über systemische Effekte der Phototherapie und Einflüsse auf das neuroendokrine Immunsystem und wie man daraus auch die bekannten Wundheilungsphänomene ableiten kann.

Dr. Wassil Nowicky stellte sein Präparat als systemischen Photosensitizer vor, welcher im Zusammenhang mit der intravenösen Lasertherapie zu sehr guten therapeutischen Ergebnissen führen kann.

Die klinischen Arbeiten zu diesem Thema wurden stets von unserem hochverehrten Kollegen Dr. Frank Andree aus San Remo vorgetragen. Er hat dazu in der Fachwelt stark beachtete Arbeiten publiziert. Ihm wurde nach seinem tragischen Tod im Frühjahr 2010 mit einer Schweigeminute gedacht.

Im nächsten Vortrag berichteten Dr. Giuseppe Vallesi und Francesco Raggi über neueste Ergebnisse in der Sportmedizin mittels der intravenösen Lasertechnik. Besonders interessant ist dies unter dem Aspekt, dass die Lasertherapie nach einem neuesten Statement der internationalen Dopingagentur nicht als Doping angesehen wird.

Im letzten Vortrag des Kongresses zeigte der Tierarzt Dr. Thomas Backhaus aus Longuich bei Trier anhand von Bluttrocknungsmustern eine neue Möglichkeit auf, den Effekt der Lasertherapie auf das Blut und überhaupt den Zustand des Körpers mit einer neuen Methode zu messen und anschaulich zu demonstrieren.

Ein Großteil der Vorträge kann ab sofort bei der EGLA auf DVD angefordert werden. 